\title{
Sistema de recomendaciones para entornos de Digital Signage soportado en un esquema de cooperación smart tv-smartphone
}

\author{
Francisco Martínez-Pabón ${ }^{*}$ \\ Víctor Garzón-Marín ${ }^{* *}$ \\ Juan Camilo Ospina-Quintero*** \\ Jhon Jairo Ibarra-Samboni**** \\ Jaime Caicedo-Guerrero ${ }^{* * * * *}$ \\ Ángela Chantre-Astaiza***** \\ Gustavo Ramírez-González
}

Recibido: 18/10/2013 • Aceptado: 17/01/2014

\begin{abstract}
Resumen
La publicidad en entornos de Digital Signage demanda el enriquecimiento de la aproximación clásica de recomendación orientada a individuos, a través de la entrega de anuncios para un grupo de personas que observa una pantalla pública. Aunque la precisión que se pueda lograr en las recomendaciones es importante, lo es aún más el grado de novedad que puedan percibir los usuarios. Este balance puede obtenerse no solo a través de la mejora de los algoritmos de recomendación, sino también a través de un adecuado esquema de cooperación multi-pantalla. En este sentido, el presente artículo propone una aproximación para la construcción de un sistema de recomendaciones para entornos de Digital Signage soportado en un esquema de cooperación Smart TV-Smartphone.
\end{abstract}

Palabras clave: sistemas de recomendaciones, publicidad ubicua, técnicas de agregación, digital signage, Smart TV

\footnotetext{
PhD(c) en Ingeniería Telemática. Investigador Grupo de Ingeniería Telemática, Universidad del Cauca, Popayán, Colombia. Calle 5 \# 4 -70, Tel: (57 2) 8209800 ext. 2127, email: fomarti@unicauca.edu.co.

** Ing. en Electrónica y Telecomunicaciones. Investigador Grupo de Ingeniería Telemática, Universidad del Cauca, Popayán, Colombia. Calle 5 \# 4 -70, Tel: (57 2) 8209800 ext. 2127, email: vgarzon@unicauca.edu.co.

*** Ing. en Electrónica y Telecomunicaciones. Investigador Grupo de Ingeniería Telemática, Universidad del Cauca, Popayán, Colombia. Calle 5 \# 4 -70, Tel: (57 2) 8209800 ext. 2127, email: jcospina@unicauca.edu.co.

**** Ing. en Electrónica y Telecomunicaciones. Investigador Grupo de Ingeniería Telemática, Universidad del Cauca, Popayán, Colombia. Calle 5 \# 4 -70, Tel: (57 2) 8209800 ext. 2127, email: jjibarra@unicauca.edu.co.

***** Ing. en Electrónica y Telecomunicaciones. Investigador Grupo de Ingeniería Telemática, Docente Universidad del Cauca, Popayán, Colombia. Calle 5 \# 4 -70 Oficina 405, Tel: (57 2) 8209800 ext. 2127, email: jcaicedo@unicauca.edu.co.

******* Magíster en Marketing. Profesora de Planta Departamento de Ciencias del Turismo, Universidad del Cauca, Popayán, Colombia. Calle 5 \# 4 -70, Tel: (57 2) 8209800 ext. 2127, email: achantre@unicauca.edu.co.

******** PhD. en Ingeniería Telemática. Profesor de Planta Departamento de Telemática, Universidad del Cauca, Popayán, Colombia. Calle 5 \# 4 -70, Tel: (57 2) 8209800 ext. 2127, email: gramirez@unicauca.edu.co.
} 


\title{
Recommendations system for Digital Signage environments based on a smart tv-smartphone cooperation scheme
}

\begin{abstract}
Advertisement in Digital Signage environments demands the enrichment of the classic approach of recommendation focused on individuals by means of the delivery of ads for a group of people observing a public screen. Even though the accuracy that can be achieved in the recommendations is important, the degree of novelty perceived by the users is even more important. This balance can be obtained not only through the improvement of the recommendation algorithms, but also through an adequate multiscreen cooperation scheme. Therefore, this paper proposes an approach for the construction of a recommendations system for Digital Signage environments based on a Smart TV-Smartphone cooperation scheme.
\end{abstract}

Keywords: recommendations systems, ubiquitous advertising, aggregation techniques, digital signage, Smart TV. 


\section{INTRODUCCIÓN}

La publicidad juega un papel transcendental en el comercio moderno, al punto que grandes compañías como Google obtienen importantes ingresos a partir de contenido publicitario. Específicamente la publicidad es parte de la promoción, una de las áreas del marketing, y es definida como "cualquier forma remunerada de presentación y promoción no personal de ideas, bienes o servicios por parte de un sponsor identificado" [1]. Actualmente, un nuevo paradigma conocido como publicidad ubicua, el cual se refiere al uso de tecnologías de computación ubicua con fines publicitarios [1], ha surgido como una interesante promesa para consumidores y anunciantes.

Pese a que la mayoría de las aproximaciones en este contexto han sido concebidas para dirigir los anuncios a los dispositivos personales de los usuarios (Smartphones, tablets, $P C$ ), aún los espacios públicos resultan muy interesantes para la industria, teniendo en cuenta que el $75 \%$ de las decisiones de compra se toma en los propios lugares de venta o cerca de ellos [2]; este campo, conocido como Digital Signage, hace referencia al despliegue de contenido digital a través de pantallas públicas [3]. Tradicionalmente, el talón de Aquiles de la publicidad ha sido la falta de contenido personalizado para los usuarios, especialmente en entornos de Digital Signage, donde la entrega de anuncios sigue una filosofía broadcast, convirtiéndose así en uno de los principales desafíos para la publicidad ubicua. En este contexto, los sistemas de recomendaciones (SR) surgen como una poderosa alternativa; este tipo de sistemas aplican técnicas de búsqueda y filtrado de información para proporcionar a los usuarios sugerencias personalizadas sobre un conjunto de ítems en un dominio específico [4].

No obstante, la entrega de recomendaciones en ambientes de Digital Signage conlleva el análisis de algunas consideraciones. En primer lugar, los anuncios deben ir dirigidos a un grupo de personas que están observando la pantalla, más que a individuos en particular; por otro lado, la precisión de las recomendaciones es una métrica que rige frecuentemente la evaluación de este tipo de sistemas, pero en los entornos publicitarios el grado de novedad que se introduce a través de la recomendación puede ser tan o más importante que la precisión misma, con fines de persuasión.

El presente trabajo propone una aproximación para la construcción de un sistema de recomendaciones en un entorno de Digital Signage, soportado en un esquema de cooperación Smart TV-Smartphone; la fase de experimentación se concentra en la evaluación de algunas técnicas de recomendación grupal, de acuerdo con parámetros de novedad y precisión. De acuerdo con lo anterior, el artículo se desarrolla a partir de la siguiente estructura: en la sección 1 se presentan algunos trabajos relacionados; la sección 2 describe brevemente la arquitectura de referencia propuesta; la sección 3 resume los resultados experimentales, que son discutidos posteriormente en la sección 4. Finalmente se presentan algunas conclusiones. 


\section{ESTADO DEL ARTE}

A medida que los entornos de computación ubicua evolucionan, surgen muchos campos de aplicación atractivos y, sin lugar a dudas, la publicidad ubicua es uno de ellos. Como fue discutido previamente, la personalización es uno de los aspectos clave en este tipo de ambientes, y en ese sentido los SR constituyen una poderosa herramienta para enfrentar este desafío. Estos sistemas han sido estudiados durante las últimas dos décadas y tradicionalmente se han considerado tres técnicas para su implementación: el filtrado colaborativo es probablemente la alternativa más usada, y consiste en computar correlaciones entre usuarios a través del reconocimiento de su afinidad en preferencias y ratings otorgados sobre un conjunto de ítems con anterioridad; por otro lado, los sistemas basados en contenido, parten del comportamiento histórico del usuario, utilizando sus preferencias para predecir ratings sobre ítems, de acuerdo con la similitud que éstos guardan con otros ítems calificados en el pasado; finalmente, los sistemas híbridos combinan dos o más técnicas para mejorar el funcionamiento del SR. En este sentido, algunos trabajos resumen los principales conceptos, algoritmos y técnicas relacionadas con este campo [5-6]. No obstante, de acuerdo con [4] "el desarrollador de un SR para un dominio de aplicación específico debería entender las diferentes características del mismo, sus requerimientos, desafíos de aplicación y limitaciones", lo cual evidencia la importancia del dominio de aplicación para sentar las bases de cualquier tipo de investigación en esta área. En consecuencia, algunos trabajos han dado los primeros pasos para caracterizar dominios de publicidad ubicua, incluyendo sus características, desafíos y algunas tecnologías clave [7-8].

Por otro lado, como fue mencionado anteriormente, dos aspectos merecen ser analizados desde la perspectiva de los entornos de Digital Signage: recomendaciones para grupos de individuos y el balance precisión/novedad. Con respecto al primero, algunas aproximaciones han sido desarrolladas para la generación de recomendaciones grupales: [9] analiza diferentes aspectos relacionados con las recomendaciones para grupos, y [10] discute algunas estrategias conocidas como técnicas de agregación, las cuales tratan de consolidar un promedio a partir de preferencias individuales con miras a la definición de un modelo grupal para la entrega de recomendaciones. Otros sistemas relacionados con esta aproximación como Polylens [11], una versión especializada de MovieLens, recomienda películas usando un algoritmo que combina listas de recomendación para usuarios individuales y las organiza en orden decreciente; MusicFX [12], por su parte, recomienda canciones para grupos de usuarios usando una suma normalizada de calificaciones recibidas para todos los ítems de la lista. Por otro lado, Intrigue [13] soporta la configuración de planes turísticos para grupos heterogéneos de usuarios a partir de modelos de preferencias de sub-grupos homogéneos de usuarios, mientras que [14] desarrolló una propuesta para ambientes de publicidad 
ubicua usando una aproximación de agregación para recomendar anuncios a grupos de personas en un gimnasio; los anuncios son desplegados en una pantalla pública y en dispositivos móviles, pero básicamente son replicados, y no existe un mecanismo de interacción entre ellos. Además, solo se consideró una técnica de agregación aditiva durante la experimentación.

En otro sentido, con respecto al despliegue de anuncios en ambientes ubicuos, algunos autores han generado reflexiones sobre consideraciones relevantes para el diseño de entornos de Digital Signage [3]. Igualmente, otros trabajos han hecho énfasis en el despliegue de anuncios para dispositivos móviles usando algunos modelos de interacción entre pantallas con tecnologías como Bluetooth o NFC [15-16]. No obstante, un esquema multi-pantalla cooperativo, donde el contenido no se replique en cada despliegue sino que se especialice en cada dispositivo, es una característica deseable que aún ha sido poco explorada en entornos publicitarios. Aunque algunas aproximaciones han considerado escenarios $n$-screen [17], específicamente la investigación sobre el potencial de un esquema de cooperación SmartTV-Smartphone es aún incipiente.

\section{ARQUITECTURA DEL SISTEMA DE RECOMENDACIONES PARA ENTORNOS DE DIGITAL SIGNAGE}

La arquitectura de referencia para el sistema de recomendaciones propuesto se muestra en la figura 1. De acuerdo con el estado del arte, los espacios de Digital Signage tradicionales usan pantallas públicas con información estática siguiendo una filosofía eminentemente broadcast; aunque algunas aproximaciones usan dispositivos móviles para proporcionar algún grado de interactividad, realmente no se considera un paradigma coperativo multi-pantalla ( $n$-screen) como tal, así que de alguna manera se termina desperdiciando la capacidad de las pantallas de los Smartphones o las Tablets.

En estos términos, la arquitectura propuesta considera un esquema de coperación Smart TV-Smartphone, de tal manera que la pantalla del televisor se comporta como una pantalla pública que recomienda anuncios para el grupo de personas que se encuentra frente a la misma, al tiempo que la pantalla de cada uno de los Smartphones despliega anuncios con un carácter más personalizado para cada uno de los usuarios. La hipótesis sobre la cual se soporta el planteamiento de este esquema es que los anuncios desplegados en los Smartphones tendrán un mayor grado de precisión que los anuncios grupales, lo cual es un factor que potencia el despliegue de anuncios novedosos a través de la pantalla pública. Al tratar de generar recomendaciones para un grupo de personas, las técnicas de agregación no serán suficientemente precisas para cada usuario en particular, pero en cambio pueden generar un conjunto de anuncios desconocidos y novedosos cuando las personas observan pantalla pública, algo valioso para entornos de publicidad ubicua con fines de persuasión. 
En consecuencia, se ha proporcionado una implementación de referencia para la arquitectura propuesta usando el framework Apache Mahout [18] para el SR, el SDK de Samsung para el Smart TV [19] y la plataforma Android para la aplicación móvil. Igualmente, se ha desarrollado un API estilo REST para habilitar la comunicación entre el $S R$, el Smart TV y los Smartphones, al tiempo que un protocolo basado en $U P n P$ fue implementado para soportar la colaboración Smart TV-Smartphone. Como una breve descripción del protocolo, la figura 2 muestra un diagrama de flujo simple relacionado con el proceso de descubrimiento entre los teléfonos y el Smart TV, y la tabla 1 muestra la estructura de un mensaje para publicar anuncios en la pantalla del $T V$ desde el móvil.

Tabla 1. Estructura del mensaje para publicar anuncios en la pantalla del TV desde el Smartphone.

\begin{tabular}{|c|c|c|}
\hline \multicolumn{3}{|c|}{ Ad post message request } \\
\hline Source-destination & \multicolumn{2}{|c|}{ Smartphone-Smart TV } \\
\hline Type & \multicolumn{2}{|l|}{ HTTP POST } \\
\hline Path & \multicolumn{2}{|c|}{ http://TV_IPADDR/ws/app/SMARTA/connect } \\
\hline Path parameters & \multicolumn{2}{|c|}{ TV_IPADDR: Smart TV IP address } \\
\hline \multirow[t]{11}{*}{ HTTP Headers } & SLDeviceID & Random sequence of 10 alphabetic characters \\
\hline & ProductID & SMARTDev \\
\hline & VendorID & VendorMe \\
\hline & msgNumber & Auto incremental sequence number for the message \\
\hline & Content-Type & application/json \\
\hline & Connection & Keep-Alive \\
\hline & Accept & $* / *$ \\
\hline & Accept-Encoding & gzip, deflate, sdch \\
\hline & Accept-Language & es,en-US; $\mathrm{q}=0.8, \mathrm{en} ; \mathrm{q}=0.6$ \\
\hline & Transfer-Encoding & chunked \\
\hline & Content-Length & Number of sent bytes in JSON format \\
\hline Content & \multicolumn{2}{|l|}{$\begin{array}{l}\text { \{ } \\
\text { “type”:"post”, } \\
\text { "title":"tttt”, } \\
\text { "content”:"cccc", } \\
\text { “img_url”:"uuu” } \\
\text { \} }\end{array}$} \\
\hline
\end{tabular}

\begin{tabular}{|c|c|c|}
\hline \multicolumn{3}{|c|}{ Ad post message reply } \\
\hline \multirow{3}{*}{ Reply codes } & 200 & Accepted connection \\
\hline & 403 & Not authorized to receive messages. \\
\hline & 404 & Unknown APP_ID. \\
\hline Reply content & \multicolumn{2}{|c|}{$\begin{array}{l}\{ \\
\text { "type":"post", } \\
\text { "posted":"true/false" }\end{array}$} \\
\hline
\end{tabular}




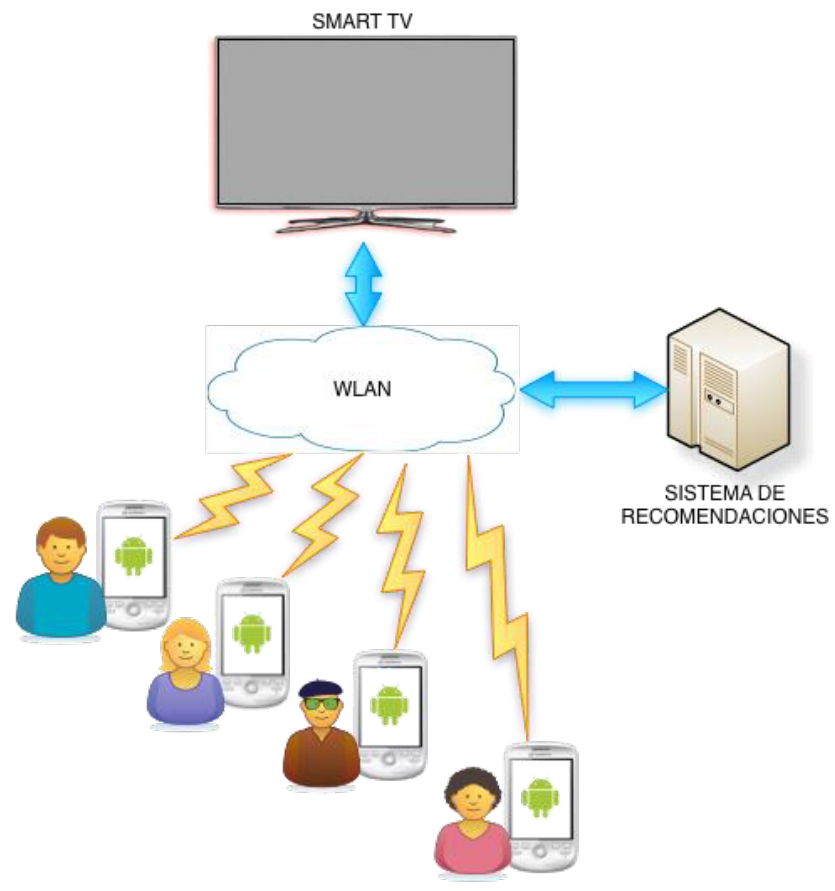

Figura 1. Arquitectura del sistema de recomendaciones con soporte Smart TV-Smartphone.

Fuente: autores

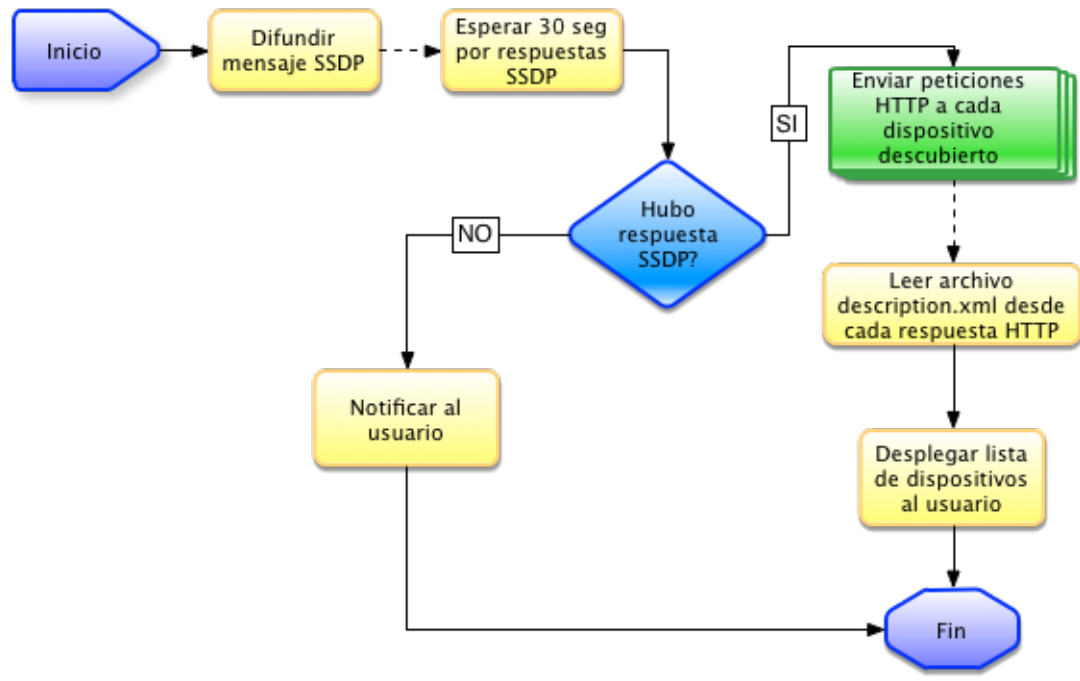

Figura 2. Proceso de descubrimiento Smart TV-Smartphone.

\section{Fuente: autores}


Tomando como base esta implementación de referencia, se ha construido un prototipo que incluye las siguientes funcionalidades: i) recomendaciones para grupos de usuarios usando técnicas de agregación; ii) recomendaciones para individuos de acuerdo con preferencias personales; iii) despliegue de recomendaciones grupales en la pantalla del televisor y recomendaciones individuales en la pantalla de los Smartphones; iv) interacción Smart TV-Smartphone para seleccionar un anuncio grupal en la pantalla del $T V$ y detallar su información en la pantalla del teléfono o publicar anuncios en el $T V$ desde el teléfono móvil. La figura 3 muestra algunas interfaces del prototipo construido.

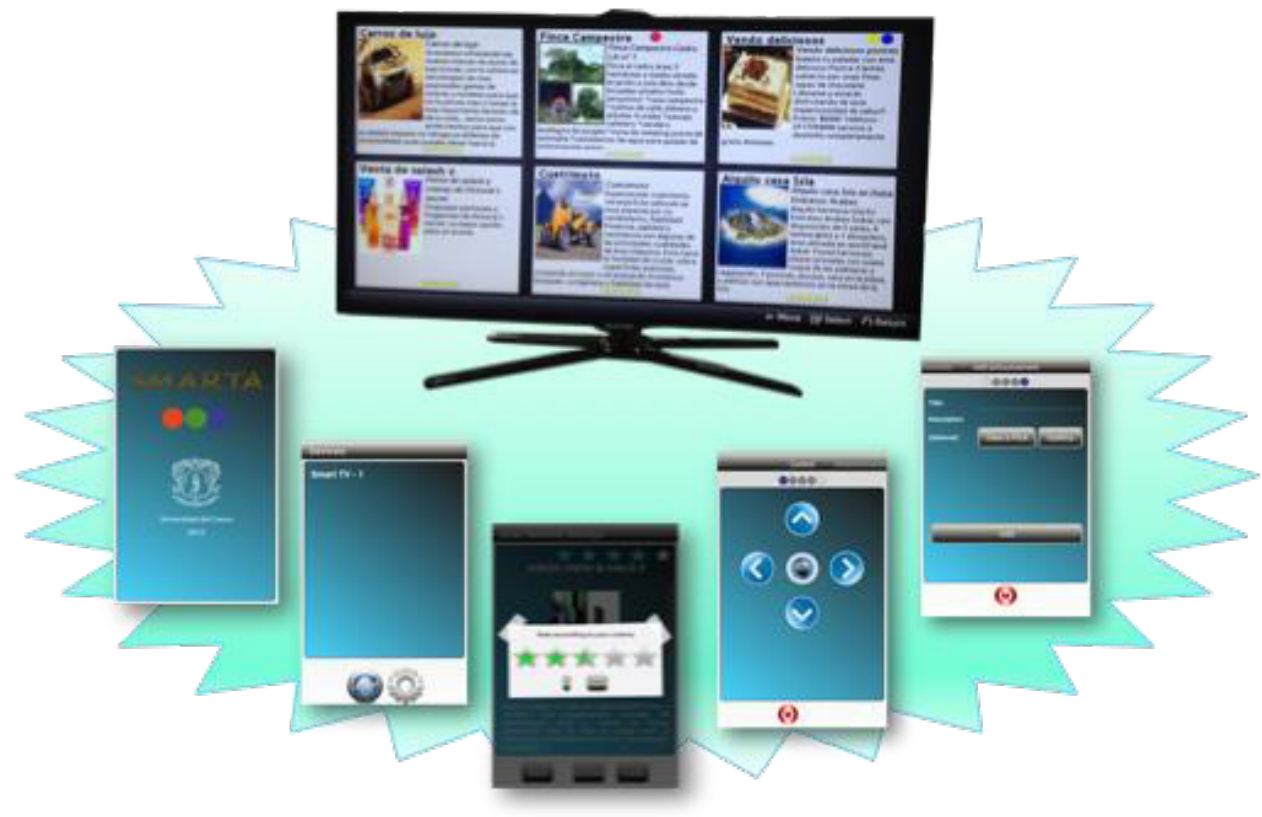

Figura 3. Interfaces del prototipo construido.

Fuente: autores

\section{RESULTADOS}

Con el ánimo de comprobar la hipótesis que sustenta el planteamiento de la arquitectura propuesta, se diseñó un marco experimental que busca evaluar el comportamiento de algunas técnicas de agregación en un contexto de Digital Signage e igualmente determinar el grado de novedad introducido, gracias a la incorporación de un esquema multi-pantalla. Este marco experimental está basado en la propuesta de [20].

\section{CONSIDERACIONES SOBRE EL DOMINIO}

Durante el experimento, se simuló un entorno de Digital Signage en un ambiente académico a través de la implementación de una cartelera electrónica de anuncios 
publicitarios, una herramienta muy popular en los campus universitarios, utilizando un esquema de interacción SmartTV-Smartphone. Por consiguiente, algunas características del dominio publicitario fueron consideradas para el diseño del experimento: la tarea principal del SR será recomendar "algunos buenos ítems"; no "todos los buenos ítems" son requeridos para propósitos publicitarios, y la maximización de la utilidad para el usuario está relacionada no solo con una buena precisión sino también con el grado de novedad introducido; incluso, en la mayoría de los casos la introducción de novedad puede ser más importante que la precisión para este contexto.

En consecuencia, aunque la tasa de falsos positivos entregada por el SR podría considerarse publicidad spam, algunos de estos anuncios podrían resultar novedosos; luego un balance entre precisión/novedad es una característica deseable. Por otro lado, la tasa de falsos negativos es particularmente relevante para los anunciantes, teniendo en cuenta sus intereses acerca de la recomendación de anuncios que se consideran importantes para sus propósitos.

\section{CONSIDERACIONES SOBRE EL DATASET}

Se construyó un dataset con información de anuncios de productos y servicios para evaluar el funcionamiento del SR en un entorno real a partir de la interacción con los usuarios. En una primera fase, un grupo de 26 estudiantes del programa de Turismo de la Universidad del Cauca incluyeron anuncios, y calificaron la mayoría de ellos usando un sistema alterno, aún sin interactuar a través del esquema Smart TV-Smartphone; el objetivo principal era construir un dataset editable y con baja dispersión para realizar pruebas offline (sin usuarios reales) sobre algunos algoritmos de recomendación, como paso previo a la evaluación de las técnicas de agregación de carácter grupal.

En una segunda fase, los tests online (con usuarios reales) incluyeron un grupo de 49 estudiantes, 21 hombres y 28 mujeres en un rango de edades entre 20 y 24 años, los cuales agregaron anuncios y calificaron algunos de ellos accediendo al $S R$ a través de la arquitectura Smart TV-Smartphone propuesta. Al final se obtuvieron 137 anuncios publicitarios y 2190 ratings para el dataset en una escala de 1 a 5 . A continuación se describirán en más detalle los aspectos considerados para las pruebas offline y online.

\section{PRUEBAS OFFLINE}

Las pruebas offline fueron diseñadas para evaluar el funcionamiento de algunos algoritmos de recomendación, particularmente técnicas de similitud siguiendo una aproximación colaborativa usuario-usuario, con el objeto de seleccionar el algoritmo con el mejor comportamiento de acuerdo con las características del dominio particular y el dataset construido. Durante esta fase, se hizo énfasis en la utilización de métri- 
cas de precisión sobre tres técnicas de similitud específicas: correlación de Pearson, correlación de Spearman y distancia euclidiana. Las métricas utilizadas para evaluar los diferentes algoritmos fueron RMSE (Root Mean Square Error), precision, recall y las curvas ROC (tabla 2). De acuerdo con los resultados obtenidos, aunque la distancia euclidiana demostró un mejor RMSE que las correlaciones de Pearson y Spearman, la correlación de Spearman mostró un mejor comportamiento de acuerdo con el análisis de las curvas de precisión, recall y ROC (Ver figura 4), razón por la cual es seleccionada como técnica de filtrado. Por otro lado, aunque el uso de la correlación de Spearman supone un costo computacional más alto que el de las otras técnicas, dado que ordena los ratings y les da una calificación previamente al cálculo de la similitud, se utilizó un mecanismo de caché disponible en Mahout para evitar recalcular valores de similitud previamente procesados.

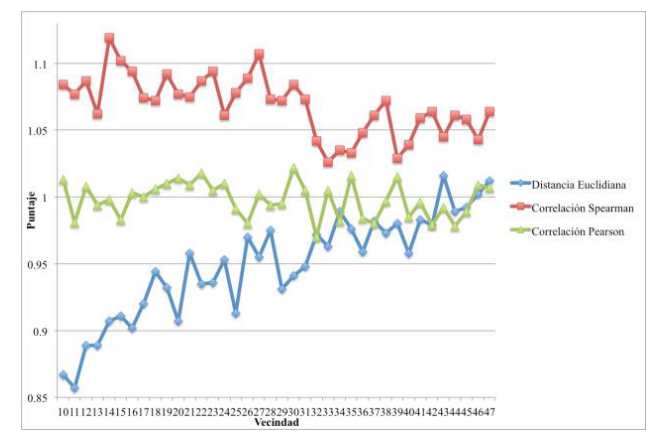

RMSE

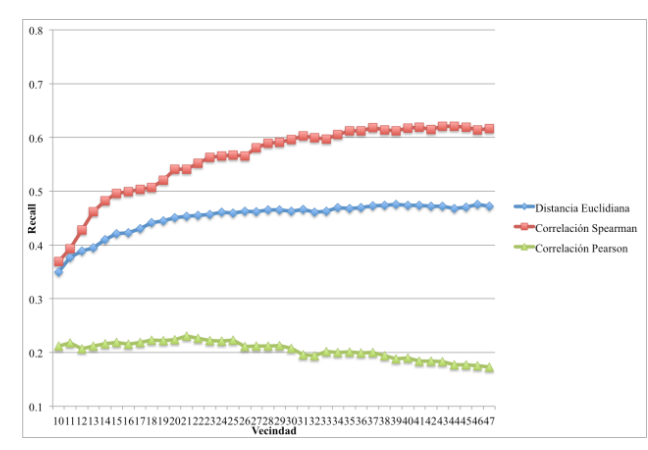

Recall

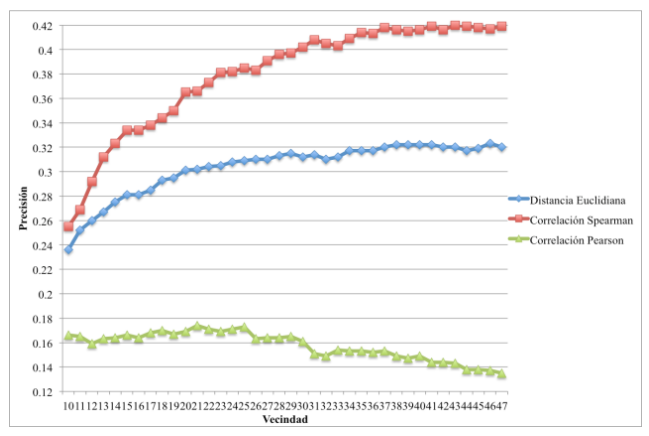

Precision

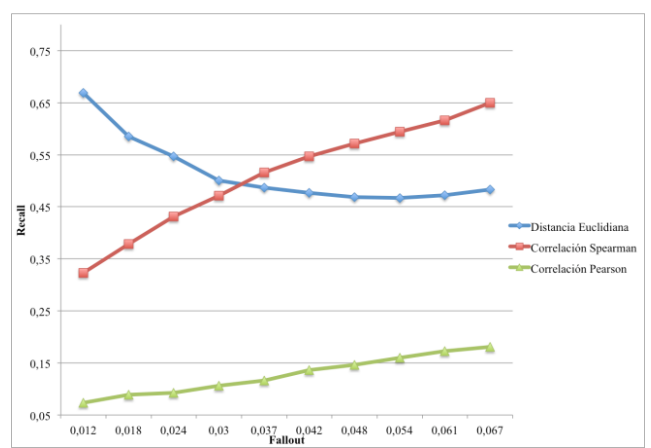

Curva ROC

Figura 4. Curvas de precisión, recall y ROC para las pruebas offline. 
Sistema de recomendaciones para entornos de Digital Signage soportado en un esquema de cooperación ... 191

Tabla 2. Métricas de evaluación para las técnicas de filtrado colaborativo.

\begin{tabular}{|l|l|l|}
\hline \multicolumn{1}{|c|}{ Tarea } & Métrica & \multicolumn{1}{c|}{ Meta } \\
\hline \multirow{2}{*}{ Predicción } & RMSE & $\begin{array}{l}\text { Calcula la desviación entre el rating que se predice y el rating real (MAE); } \\
\text { posteriormente este resultado se eleva al cuadrado para dar más peso a los } \\
\text { errores de mayor magnitud. }\end{array}$ \\
\hline \multirow{3}{*}{ Recomendación } & Precision & Probabilidad de que un ítem seleccionado sea relevante. \\
\cline { 2 - 3 } & Recall & Probabilidad de que un ítem relevante sea seleccionado. \\
\cline { 2 - 3 } & $\begin{array}{l}\text { Curvas } \\
\text { ROC }\end{array}$ & $\begin{array}{l}\text { Hacen énfasis en calcular la proporción de ítems que no son relevantes y } \\
\text { que finalmente son recomendados. }\end{array}$ \\
\hline
\end{tabular}

Fuente: Herlocker, et.al [5]

\section{PRUEBAS ONLINE}

Luego de la consolidación de los resultados de las pruebas offline, se realizó el montaje de un entorno de Digital Signage simulado en un espacio académico, usando el esquema de interacción Smart TV-Smartphone. En una primera instancia, cada individuo calificó algunos anuncios seleccionados de forma aleatoria a través de una página Web alternativa, con el objeto de minimizar el problema de cold start, una deficiencia común en los SR que hace referencia a las restricciones del sistema para generar recomendaciones cuando no se tiene suficiente información disponible, algo frecuente en el caso de usuarios o ítems nuevos.

Posteriormente, se configuraron aleatoriamente grupos de cuatro personas para interactuar con el Smart TV usando la aplicación Android en el Smartphone durante un período de aproximadamente 5 minutos. Al final, cada usuario llenó una encuesta en la cual se indagó directamente sobre el grado de novedad y precisión percibido por cada uno de los participantes con respecto a las recomendaciones entregadas tanto por el Smart TV como por el Smartphone.

Específicamente, los tests realizados consideraron la evaluación de tres técnicas de agregación seleccionadas a partir del trabajo de Masthoff [10], de acuerdo con su conveniencia y grado de aplicación en entornos publicitarios; la tabla 3 resume las definiciones de las técnicas empleadas. En este sentido, cada una de las estrategias de agregación fue sometida a prueba usando el algoritmo de similitud usuario-usuario seleccionado durante la fase de pruebas offline. De acuerdo con los resultados, en términos generales se puede concluir que las personas percibieron mayor novedad en los anuncios desplegados en la pantalla del Smart TV, como era de esperarse de acuerdo con el planteamiento de la hipótesis. No obstante, un análisis más profundo será presentado en la siguiente sección con el objeto de aclarar detalles del comportamiento de las técnicas de agregación consideradas. 
Tabla 3. Descripción de las técnicas de agregación grupal.

\begin{tabular}{|l|l|l|}
\hline $\begin{array}{c}\text { Test } \\
\text { No. }\end{array}$ & \multicolumn{1}{|c|}{$\begin{array}{c}\text { Técnica de } \\
\text { agregación }\end{array}$} & \multicolumn{1}{c|}{ Descripción } \\
\hline 1 & Aditiva & $\begin{array}{l}\text { Los ratings son adicionados. Los valores de suma mayores configuran la lista de } \\
\text { recomendación. }\end{array}$ \\
\hline 2 & Mínima miseria & $\begin{array}{l}\text { Hace una lista de los ratings con el mínimo de los ratings individuales; los ítems } \\
\text { son recomendados de acuerdo a los ratings de la lista, el más alto primero. El } \\
\text { grupo están tan satisfecho como su miembro menos satisfecho. }\end{array}$ \\
\hline 3 & $\begin{array}{l}\text { Máximo } \\
\text { beneficio }\end{array}$ & $\begin{array}{l}\text { Hace una lista de los ratings con el máximo de los ratings individuales; los ítems } \\
\text { son recomendados de acuerdo a los ratings de la lista, el más alto primero }\end{array}$ \\
\hline
\end{tabular}

Fuente: Masthoff [10]

\section{DISCUSIÓN}

La tabla 4 resume algunos resultados estadísticos con respecto a la novedad percibida por los usuarios para cada una de las técnicas de agregación. El valor de la media sugiere que la técnica de "Mínima miseria" ofrece un mejor comportamiento con respecto a la novedad percibida. Sin embargo, es importante averiguar si esta diferencia es significativa, dado que la evaluación se llevó a cabo para muestras de usuarios diferentes; en otras palabras, el propósito es determinar si el grado de novedad percibida usando la técnica de "mínima miseria" es significativamente mayor con respecto a la técnica "aditiva" o de "máximo beneficio". Para soportar este análisis, se hizo uso de la técnica estadística two tample $t$, de acuerdo con los siguientes lineamientos: se debe considerar una hipótesis nula durante la prueba; en este caso, existen dos hipótesis nulas; la primera de las cuales propone que la novedad percibida a partir del uso de la técnica aditiva es la misma con respecto a la técnica de mínima miseria; en los mismos términos, la segunda hipótesis involucra las técnicas de mínima miseria y máximo beneficio. En ambos casos, se considera una hipótesis alternativa, la cual propone que la estrategia de mínima miseria ofrece un mayor grado de novedad percibida. La tabla 5 muestra los resultados del análisis para ambas hipótesis tomando como base un valor $\mathrm{p}$ de 5 $\%$; en este sentido, un valor de p por debajo de 0,05 es una evidencia estadística que aprueba la hipótesis alternativa sobre cualquiera de las hipótesis nulas.

Tabla 4. Nivel de novedad percibido (pruebas online).

\begin{tabular}{|l|c|c|c|}
\hline \multicolumn{1}{|c|}{ Técnica de agregación } & Tamaño de muestra & Media & Desviación estándar \\
\hline Additive & 16 & $\mathrm{uA}=3,438$ & 1,209 \\
\hline Less misery & 18 & $\mathrm{uLM}=3,944$ & 0,802 \\
\hline Most pleasure & 14 & $\mathrm{uMP}=3,214$ & 0,699 \\
\hline
\end{tabular}

Fuente: autores

Revista Ingenierías Universidad de Medellín 
Tabla 5. Resultados de la prueba 2 sample t para novedad percibida.

\begin{tabular}{|c|c|c|c|}
\hline Test & Hipótesis nula & Hipótesis alternativa & Valor $p$ \\
\hline 1 & $\mathrm{uLM}=\mathrm{uA}$ & $\mathrm{uLM}>\mathrm{uA}$ & 0,084 \\
\hline 2 & $\mathrm{uLM}=\mathrm{uMP}$ & $\mathrm{uLM}>\mathrm{uMP}$ & 0,005 \\
\hline
\end{tabular}

Fuente: autores

De acuerdo con lo anterior, aunque no fue posible concluir si existe una diferencia contundentemente significativa sobre la estrategia aditiva, es claro que el grado de novedad percibido a través de la estrategia de mínima miseria es mayor que el que se logra a través de la técnica de máximo beneficio. La explicación puede estar relacionada con la naturaleza misma del funcionamiento de las técnicas: mientras la técnica de mínima miseria busca de alguna manera la satisfacción de "todos los usuarios", es posible que la estrategia de "máximo beneficio" ofrezca algunos anuncios interesantes para un usuario en particular, pero estos resultan poco o nada atractivos para otros individuos, lo cual afecta la precisión pero también la novedad percibida. Los anuncios inútiles no pueden considerarse necesariamente novedosos, así que debe buscarse un balance entre novedad y precisión.

En consecuencia, los argumentos anteriores se pueden soportar a través de un análisis similar con respecto a la satisfacción percibida. Como se puede observar en las tablas 6 y 7 , en ambos experimentos los valores obtenidos para $p$ fueron inferiores a 0,05 , lo cual significa que hay una diferencia significativa en la satisfacción percibida por los usuarios cuando se emplea la técnica de mínima miseria sobre cualquiera de las otras dos estrategias; luego se logra un mejor balance novedad/precisión.

Tabla 6. Nivel de satisfacción percibido (pruebas online).

\begin{tabular}{|l|c|c|c|}
\hline Técnica de agregación & Tamaño de muestra & Media & Desviación estándar \\
\hline Additive & 16 & $\mathrm{uA}=3,375$ & 0,957 \\
\hline Less misery & 18 & $\mathrm{uLM}=4,056$ & 0,725 \\
\hline Most pleasure & 14 & $\mathrm{uMP}=3,5$ & 0,941 \\
\hline
\end{tabular}

Fuente: autores

Tabla 7. Resultados de la prueba 2 sample t para satisfacción percibida.

\begin{tabular}{|c|c|c|c|}
\hline Test & Hipótesis nula & Hipótesis alternativa & Valor $p$ \\
\hline 1 & $\mathrm{uLM}=\mathrm{uA}$ & $\mathrm{uLM}>\mathrm{uA}$ & 0,014 \\
\hline 2 & $\mathrm{uLM}=\mathrm{uMP}$ & $\mathrm{uLM}>\mathrm{uMP}$ & 0,040 \\
\hline
\end{tabular}

Fuente: autores 


\section{CONCLUSIONES}

Una de las principales contribuciones de este trabajo es la definición de una aproximación para generar recomendaciones de anuncios en un entorno de Digital Signage, no solo abordando una mejora en los algoritmos de recomendación como tradicionalmente se ha hecho en las investigaciones de este tipo, sino apoyando el trabajo de los algoritmos para la entrega de los anuncios a través de un esquema de colaboración multi-pantalla Smart TV-Smartphone. Frecuentemente, la precisión de las recomendaciones ha sido una preocupación constante para los investigadores, pero el esquema propuesto favorece en su lugar un balance entre la precisión y la novedad de las mismas, algo indiscutiblemente valioso para un dominio de aplicación como la publicidad.

Por otro lado, las técnicas de agregación grupal de mínima miseria y máximo beneficio demostraron un mejor comportamiento que la estrategia aditiva con respecto a la novedad percibida por parte de los usuarios, pero la técnica de mínima miseria parece ofrecer un mayor grado de satisfacción, lo cual significa un mejor balance precisión/novedad para los propósitos de la investigación.

Finalmente, a manera de trabajo futuro se requiere un análisis más profundo del comportamiento de las técnicas de agregación con el ánimo de cuantificar el grado de novedad; adicionalmente, la inclusión de información contextual en los algoritmos de recomendación resulta interesante para los propósitos del dominio de aplicación.

\section{AGRADECIMIENTOS}

El presente trabajo fue soportado por la Universidad del Cauca a través de los proyectos VRI 3593 "SMARTA: Modelo para el despliegue de publicidad en entornos de computación ubicua soportado en un esquema de cooperación Smart TV - Smartphone" y VRI 4045 "MANTISS: Modelo para la adaptación de contenidos publicitarios en entornos n-screen interactivos soportados en un esquema de colaboración Smart TV - Smartphone". Francisco Martínez cuenta con la financiación del programa de becas doctorales 2012 de Colciencias, convocatoria 567.

\section{REFERENCIAS}

[1] J. Müller, F. Alt, and D. Michelis, "Pervasive Advertising," en Pervasive Advertising, J. Müller, F. Alt, and D. Michelis, eds., pp. 1-29, Springer London, 2011.

[2] C. Bauer and S. Spiekermann, "Conceptualizing Context for Pervasive Advertising," en Pervasive Advertising, J. Müller, F. Alt, and D. Michelis, eds., pp. 159-183, Springer London, 2011.

[3] U. Stalder, "Digital Out-of-Home Media: Means and Effects of Digital Media in Public Space," en Pervasive Advertising, J. Müller, F. Alt, and D. Michelis, eds. , pp. 31-56, Springer London, 2011. 
[4] F. Ricci, L. Rokach, and B. Shapira, "Introduction to Recommender Systems Handbook," en Recommender Systems Handbook, F. Ricci, L. Rokach, B. Shapira, and P. B. Kantor, eds. , pp. 1-35, Springer US, 2011.

[5] J. L. Herlocker, J. A. Konstan, A. Borchers, and J. Riedl, "An Algorithmic Framework for Performing Collaborative Filtering," presentado en Proceedings of the 22Nd Annual International ACM SIGIR Conference on Research and Development in Information Retrieval, New York, 1999.

[6] Q. Li and B. M. Kim, "An approach for combining content-based and collaborative filters," presentado en Proceedings of the sixth international workshop on Information retrieval with Asian languages - Volume 11, Stroudsburg, 2003,

[7] K. Partridge and B. Begole, “Activity-Based Advertising," en Pervasive Advertising, J. Müller, F. Alt, and D. Michelis, eds. , pp. 83-101, Springer London, 2011.

[8] P. van Waart, I. Mulder, and C. de Bont, "Meaningful Advertising," in Pervasive Advertising, J. Müller, F. Alt, and D. Michelis, eds., pp. 57-81 Springer London, 2011.

[9] A. Jameson, "More than the sum of its members: challenges for group recommender systems," presentado en Proceedings of the working conference on Advanced visual interfaces, New York, 2004.

[10] J. Masthoff, "Group Modeling: Selecting a Sequence of Television Items to Suit a Group of Viewers,” User Model. User-Adapt. Interact., vol. 14, no. 1, pp. 37-85, 2004.

[11] M. O’Connor, D. Cosley, J. A. Konstan, and J. Riedl, "PolyLens: A Recommender System for Groups of Users," en ECSCW 2001, W. Prinz, M. Jarke, Y. Rogers, K. Schmidt, and V. Wulf, eds., pp 199-218, Springer Netherlands, 2002.

[12] J. F. McCarthy and T. D. Anagnost, "MusicFX: an arbiter of group preferences for computer supported collaborative workouts," presentado en Proceedings of the 1998 ACM conference on Computer supported cooperative work, New York, 1998.

[13] L. Ardissono, A. Goy, G. Petrone, M. Segnan, and P. Torasso, "Intrigue: Personalized recommendation of tourist attractions for desktop and hand held devices," Appl. Artif. Intell., vol. 17, no. 8-9, pp. 687-714, 2003.

[14] B. D. Carolis, "Adapting News and Advertisements to Groups," en Pervasive Advertising, J. Müller, F. Alt, and D. Michelis, eds. , pp. 227-246, Springer London, 2011.

[15] R. Jose, N. Otero, S. Izadi, and R. Harper, "Instant Places: Using Bluetooth for Situated Interaction in Public Displays,” IEEE Pervasive Comput., vol. 7, no. 4, pp. 52-57, 2008.

[16] J. Ibarra et al., Integración de pizarras interactivas de bajo costo con un LMS. Propuesta para el caso .LRN - Universidad del Cauca. USA: R\&I Book, 2012, 208p.

[17] P. Santos, F. R. Ribeiro, and J. Metrolho, "Using pervasive computing technologies to deliver personal and public ads in public spaces," presentado en 2012 7th Iberian Conference on Information Systems and Technologies (CISTI), 2012.

[18] Apache Mahout: Scalable machine learning and data mining. [En linea]. Acceso: 05 de Julio, 2013; Disponible: http://mahout.apache.org/ 
[19] Samsung, Samsung Smart TV Apps Developer Forum. [En linea]. Acceso: 31 de Enero,2014; Disponible: http://www.samsungdforum.com/Devtools/Spec.

[20] J. L. Herlocker, J. A. Konstan, L. G. Terveen, and J. T. Riedl, "Evaluating collaborative filtering recommender systems," ACM Trans Inf Syst, vol. 22, no. 1, pp. 5-53, 2004. 